\title{
The BT-Settl Model Atmospheres for Stars, Brown Dwarfs and Planets
}

\author{
F. Allard \\ Centre de Recherche Astrophysique de Lyon, UMR 5574, CNRS, \\ Université de Lyon, École Normale Supérieure de Lyon, \\ 46 Allée d'Italie, F-69364 Lyon Cedex 07, France \\ email: fallard@ens-lyon.fr
}

\begin{abstract}
We present a grid of stellar and substellar atmosphere models covering the range from solar-mass stars to the latest-type $\mathrm{T}$ and $\mathrm{Y}$ dwarfs with a single setup. For the first time our synthetic spectra and photometry reproduce the formation of clouds and in particular their clearing at the L/T transition. The BT-Settl models also naturally explain the dustier infrared properties of planets as an effect of low surface gravity.
\end{abstract}

Keywords. brown dwarfs, planets, clouds, etc.

As brown dwarfs and imaged planets are being discovered, they reveal their spectral and/or photometric properties. Late $\mathrm{M}$ type and L type dwarfs see the onset of silicate dust formation which exerts a green house effect in their atmospheres, making them look red in the near-IR bandpasses $(J-K)$. At optical wavelengths cloud formation has the effect of causing the sedimentation of important opacity sources ( $\mathrm{TiO}, \mathrm{VO}, \mathrm{CaH}$, etc.) while dust scattering depresses the optical pseudo-continuum. As the cloud layers sink below the photosphere in $\mathrm{T}$ type dwarfs, the optical becomes dominated by alkali absorption lines and methane bands begin to form in the $W 1$ and $K$ bandpasses, as well as ammonia bands in the $H$ and $W 3$ bandpass. Water ice condensation affects $\mathrm{Y}$ type brown dwarfs, where the strength of water and methane bands leaves only flux escape in narrow windows of minimum opacity, and where this important line blanketing causes flux to be redistributed into the $4.5 \mu \mathrm{m}$ peak that becomes as bright as the near-IR flux peaks. The imaged planets have, to the greater surprise of the community, redder near-IR colors then L type brown dwarfs.

Many modelers have attempted to explain this behavior. We present in this paper our explanation using a parameter-free cloud model which takes into account all important micro processes (supersaturation, nucleation, condensation, coalescence, coagulation, and mixing) according to Rossow(1978) and to Radiation Hydrodynamical (RHD) simulations (Freytag et al. 2010). Fig. 1 shows this transformation of the spectral properties of these objects, where we have overlaid the latest version of the BT-Settl model atmospheres (Allard et al. 2012) using this parameter-free cloud model. We find in general a good agreement of the models to the ground-based and Spitzer observations across the sequence. We find that reducing the gravity to correspond to the young planets allows a good reproduction of their near-IR spectral energy distribution.

These results imply that there is no fundamental difference between planetary and brown dwarf spectral properties, at least for the candidates compared in Fig. 1. Beta Pic b shows slightly different optical colors which could be explained by the reflection of the stellar light on clouds. The current BT-Settl models do not generate important reflection effects due to the silicate nature of the clouds predicted for this planet. More 


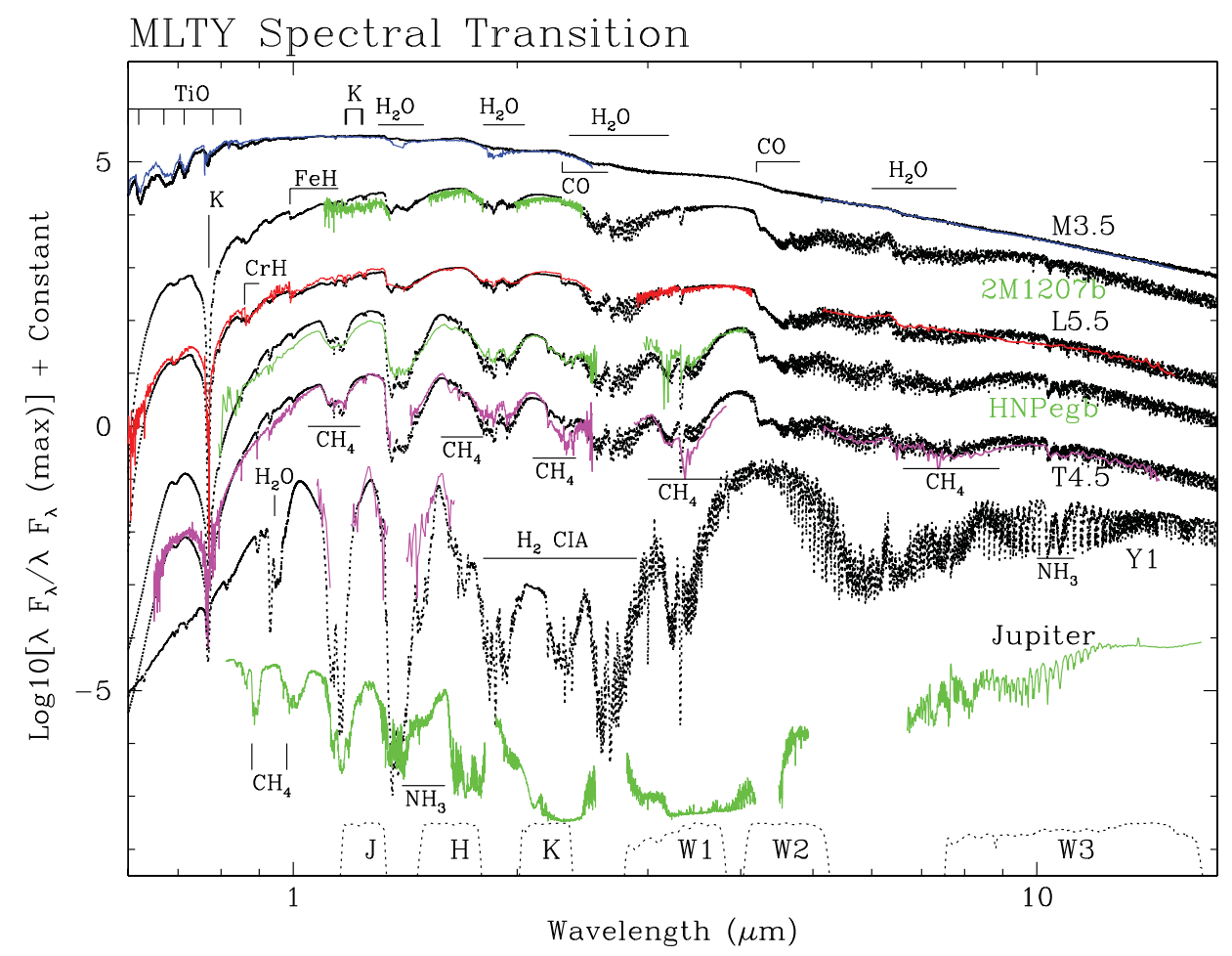

Figure 1. The SED of the M dwarf star Gl101A (first curve from the top), the L dwarf 2MASS 1507-16 and the T dwarf 2MASSJ 0559-14 using the United Kingdom Infrared Telescope (UKIRT) and Spitzer (among other) spectroscopic observations (Leggett et al. 2002, Geballe et al. 2002, Cushing et al. 2008), and the Y1 dwarf WISE 0350-5658 (Kirkpatrick et al. 2012) (second and third curves from the bottom), as well as those of the planetary mass objects 2M1207b (Chauvin et al. 2005), HNPegb (Luhman et al. 2007) and Jupiter (first fourth and sixth curves from the bottom) using the medium-resolution infrared spectrograph SpeX of the NASA InfraRed Telescope Facility (IRTF) on Mauna Kea, SINFONI and Spitzer spectroscopic spectra (Patience et al. 2012, Leggett et al. 2008), and the infrared spectrum of Jupiter obtained with the Visual and Infrared Mapping Spectrometer (VIMS) onboard Cassini. The BT-Settl models (dotted curves) are shown for $\left[T_{\text {eff }}, \log g\right]=[3000 \mathrm{~K}, 5.0],[1600 \mathrm{~K}, 5.0],[1600 \mathrm{~K}, 5.5]$, $[1200 \mathrm{~K}, 5.5],[1200 \mathrm{~K}, 5.5]$, and [450 K, 5.0] from top to bottom. Also shown are the Mauna Kea Observatories (MKO) and WISE filter bandpasses in the lower part of the plot (dotted lines).

reflective salts or ices clouds could however if present cause increased flux at optical wavelengths.

\section{References}

Allard, F., Homeier, D., Freytag, B., \& Sharp, C. M. 2012, EAS Publications Series, 57, 3

Chauvin, G., Lagrange, A.-M., Dumas, C., et al. 2005, A\&A (Letters), 438, L25

Cushing, M. C., Marley, M. S., Saumon, D., et al. 2008, ApJ, 678, 1372

Freytag, B., Allard, F., Ludwig, H.-G., Homeier, D., \& Steffen, M. 2010, A\&̈A, 513, A19

Geballe, T. R., Knapp, G. R., Leggett, S. K., et al. 2002, ApJ, 564, 466

Kirkpatrick, J. D., Gelino, C. R., Cushing, M. C., et al. 2012, ApJ, 753, 156

Leggett, S. K., Golimowski, D. A., Fan, X., et al. 2002, ApJ, 564, L452

Leggett, S. K., Saumon, D., Albert, L., et al. 2008, ApJ, 682, 1256

Luhman, K. L., Patten, B. M., Marengo, M., et al. 2007, ApJ, 654, 570

Patience, J., King, R. R., De Rosa, R. J., et al. 2012, A\&A, 540, A85

Rossow, W. B. 1978, Icarus, 36, 1 